\title{
SOME NEW INTEGRAL INEQUALITIES WITH APPLICATIONS
}

\author{
KE Ding, Jong-KyU KIM AND NAN-JING HuANG
}

Abstract. In this paper, we introduce and study some new integral inequalities in one variable and two independent variables which provide explicit bounds on unknown functions, and apply these integral inequalities to study the qualitative behavior of the solution for a partial differential equation and an integral equation, respectively.

Mathematics subject classification (2000): 26D15, 34A12.

Key words and phrases: integral inequality, two independent variables, partial differential equation, integral equation.

\section{REFERENCES}

[1] D. Bainov, P. Simeonov, Integral Inequalities and Applications, Kluwer Academic Publishers, Dordrecht, 1992.

[2] Y. H. KIM, T. M. Rassias, Generalizations of Hardy integral inequality, Nonlinear Funct. Anal. Appl. 7, (2002), 455-463.

[3] O. LipovAn, A retarded integral inequality and its applications, J. Math. Anal. Appl., 285, (2003), 436-443.

[4] M. MEDVED, Nonlinear singular integral inequalities for functions in two and $n$ independent variables, J. Inequal. Appl., 5, (2000), 287-308.

[5] F. W. MenG, W. N. LI, On some new integral inequalities and their applications, Appl. Math. Comput., 148, (2004), 381-392.

[6] B. G. PACHPATTE, Inequalities for Differential and Integral Equations, Academic Press, New York, 1998.

[7] B. G. PACHPATTE, On some fundamental integral inequalities and their discrete analogues, J. Ineqaul. Pure Appl. Math., 2, (2001), article 15.

[8] T. M. RASSIAS (ed), Survey on Classical Inequalities, Mathematics and its Applications, 517, Kluwer Academic Publishers, Dordrecht, 2000.

[9] Y. G. Sun, On retarded integral inequalities and their applications, J. Math. Anal. Appl., 301, (2005), 265-275. 